\title{
Com Um Brilhozinho nos Olhos: Três experiências promotoras de ensino do pensamento crítico na área da Matemática
}

\author{
Maria Manuel Nascimento, Paula Catarino, \\ Eva Morais \& Paulo Vasco
}

\begin{abstract}
Resumo:
A sociedade atual do conhecimento requer cidadãos preparados para o mundo que os rodeia e conscientes das decisões que têm que tomar no seu dia a dia. Neste sentido, vários estudos referem que o pensamento crítico é essencial no mercado de trabalho, sendo recomendado que esta competência esteja integrada na formação do aluno do ensino superior. O presente trabalho surge como resultado desta preocupação na área da Matemática, tendo por base as mudanças efetuadas após o curso de formação de professores em Educação para o Pensamento Crítico (PC) do projeto Erasmus+ CRITHINKEDU. Trata-se de um trabaIho de natureza qualitativa e descritiva, no qual se relatam três estudos de caso de diferentes unidades curriculares modificadas depois do curso de formação. São apresentadas as modificações, comparativamente ao ano letivo anterior, ao nível da planificação, das estratégias de ensino e de aprendizagem utilizadas, e da avaliação da aprendizagem. Através dos resultados obtidos conclui-se que há ainda trabalho a desenvolver no sentido de integrar as orientações preliminares para a qualidade na Educação do PC propostas no âmbito do projeto CRITHINKEDU nas práticas letivas descritas, nomeadamente as da avaliação.
\end{abstract}

Palavras-chave:

pensamento crítico; ensino superior; matemática; álgebra linear; estatística. 


\title{
With a Little Brightness in the Eyes: Three experiences of critical thinking inclusion in mathematic courses
}

\begin{abstract}
Today's knowledge society requires citizens prepared for the world challenges and aware of the decisions they have to make in their day-to-day lives. In this sense, several studies indicate that critical thinking is essential in the labor market recommending that it be integrated in higher education. The present work arises from this concern in the Mathematics field. It is based on the changes fostered by the teacher-training course in Education for Critical Thinking (CT) of the Erasmus+ CRITHINKEDU project. This is a qualitative and descriptive work, in which three case studies of different curricular units were modified after the training course. The changes, compared to the previous academic year, are presented at the level of planning, teaching and learning strategies used, as well as the learning assessment. Through the results obtained, we conclude that there is still work to be done in order to integrate the preliminary orientations for quality in the CT Education, proposed in the framework of the CRITHINKEDU project, in the teaching practices described, namely those concerned with assessment.
\end{abstract}

Keywords: critical thinking; higher education; mathematics; linear algebra; statistics.

\section{Avec une petite clarté dans les yeux: Trois expériences d'inclusion de la pensée critique en mathématiques}

Résumé: La société de la connaissance actuelle exige que les citoyens soient préparés pour le monde qui les entoure et qu'ils soient conscients des décisions à prendre dans leur vie quotidienne. En ce sens, plusieurs études indiquent que la pensée critique est essentielle sur le marché du travail et qu'elle doit être intégrée dans la formation des étudiants de l'enseignement supérieur. Ce présent travail découle de cette préoccupation dans le domaine des mathématiques, et se base sur les changements promus par le cours de formation des enseignants en Éducation à la pensée critique (PC) du projet Erasmus+ CRITHINKEDU. II s'agit d'un travail qualitatif qui décrit trois études de cas de disciplines qui ont été modifiées après la formation. Les changements introduits, comparés avec l'année académique précédente, sont présentés au niveau de la planification, des stratégies d'enseignement et d'apprentissage utilisées et de l'évaluation de l'apprentissage. Les résultats obtenus permettent de conclure qu'il reste encore du travail à faire pour intégrer dans les pratiques pédagogiques décrites les orientations préliminaires en matière de qualité de la PC dans l'Éducation, proposées dans le cadre du projet CRITHINKEDU, surtout ceux de l'évaluation.

Mots clés: pensée critique; enseignement supérieur; mathématique; algèbre linéaire; statistique.

\section{Con un brillo en los ojos: Tres experiencias de inclusión del pensamiento crítico en las matemáticas}

Resumen: Hoy en día, la sociedad del conocimiento requiere que los ciudadanos se preparen para los desafíos del mundo y que sean conscientes de todas las decisiones que toman en el día a día. En este sentido, varios estudios refieren que el pensamiento crítico (PC) es esencial en el mercado de trabajo, siendo recomendado que esta competencia esté integrada en la formación del alumno en la enseñanza superior. El presente trabajo surge como resultado de esta preocupación en el área de las Matemáticas, teniendo como base las modificaciones promovidas por e curso de formación de profesores en Educación para el Pensamiento Crítico del proyecto Erasmus+ CRITHINKEDU. Se trata de un trabajo de naturaleza cualitativa y descriptiva, en el cual se relatan tres estudios de caso de unidades curriculares que fueron modificadas después del curso de formación. Se presentan las modificaciones, en relación con el año lectivo anterior, al nivel de la planificación, de las estrategias de enseñanza y de aprendizaje utilizadas, y de la evaluación del aprendizaje. A través de los resultados obtenidos se concluye que todavía hay trabajo por desarrollar en el sentido de integrar en las prácticas lectivas descritas las orientaciones preliminares para la calidad en la Educación del PC, propuestas en el marco del proyecto CRITHINKEDU, sobre todo las de la evaluación.

Palabras-clave: pensamiento crítico; enseñanza superior; matemática; álgebra lineal; estadística. 


\section{Introdução e contextualização}

A educação tem um papel preponderante na preparação dos jovens para a sua plena integração na sociedade e é um desafio para uma sociedade em constante mutação. Em particular, as Instituições de Ensino Superior (IES) devem procurar encontrar estratégias que permitam aos alunos desenvolver o Pensamento Crítico (PC) que os ajudarão a enfrentar o mundo laboral e as exigências que a sociedade atual Ihes impõe.

Não sendo unânimes as várias definições de PC propostas por diferentes autores deste campo de investigação, adotamos a definição de Facione (1990, p. 2), que entende que

o pensamento crítico é um julgamento propositado e autorregulador que resulta na interpretação, análise, avaliação e inferência, bem como na explicação das considerações conceituais, metodológicas, criteriológicas ou contextuais sobre as quais esse julgamento se baseia (...).

O conceito de Facione foi adotado porque o PC é representado, não apenas pelo uso sistemático e intencional das diferentes formas de pensamento racional, mas também porque, de forma explícita, os indivíduos têm que participar nesse processo de construção do seu pensamento. As competências (interpretação, análise, inferência, avaliação, explicação, autorregulação) e as disposições (procura da verdade, mente aberta, mente analítica, sistematicidade, autoconfiança, curiosidade, intelectual, maturidade) do PC contribuem para o sucesso dos alunos e facilitam a transição para o mercado de trabalho em todas as áreas profissionais, daí a necessidade de serem abordadas (Dominguez, 2018a). O PC é valorizado pelas empresas, sendo considerado como uma das soft skills essenciais nas áreas profissionais (Dominguez, 2018a). No trabalho de Cruz, Nascimento, Payan, Dominguez, Silva e Morais (2017) as competências do PC foram mencionadas e consideradas importantes em focus group, nomeadamente em Saúde e STEM (Science, Technology, Engineering, and Mathematics, trabalho apresentado em Dominguez, 2018a).

Na perspetiva do ensino da matemática, e de acordo com Huang, Ricci e Mnatsakanian (2016), os alunos têm a capacidade de melhorar e desenvolver o seu PC quando aprendem matemática ao resolver problemas (matemáticos), identificando possíveis soluções e avaliando e justificando as suas razões para os resultados e, assim, adquirindo confiança na forma como pensam. Huang et al. (2016) ainda referem que o PC, aliado ao raciocínio matemático, permite aos alunos refletir sobre o seu próprio raciocínio, pois devem ser ensinados a: identificar cenários; avaliá-los; selecionar estratégias de resolução de problemas; identificar possíveis conclusões que têm que ser lógicas; descrever e resumir uma solução; e, por vezes, indicar como essas soluções se aplicarão a problemas matemáticos mais avançados. 
Nas IES, se os professores ensinarem matemática integrando estratégias promotoras do PC, os alunos serão capazes de encontrar as informações necessárias, avaliar as informações, resolver problemas (Jacob, 2012) e melhorar a sua comunicação. Também serão capazes de tomar decisões adequadas para conseguirem resolver problemas de forma independente, sistemática e lógica (Jacob, 2012; Huang et al. 2016). Assim, o papel do professor deve centrar-se no desenho da aula de matemática que promova o PC e a metacognição para a vida (Huang et al., 2016). Sendo a matemática uma área transversal a muitos domínios do conhecimento (e.g., STEM, Ciências Humanas) deve ser vista como base para o desenvolvimento e para a transferência de PC para inúmeras profissões e situações do dia a dia onde a matemática está presente e nas IES através das respetivas disciplinas.

No sentido de integrar de uma forma mais sistemática o ensino do PC nos curricula das IES europeias, o projeto "Critical Thinking Across the European Higher Education Curricula - CRITHINKEDU" identificou as competências e disposições do PC mais procuradas pelos empregadores em diferentes áreas profissionais e procedeu à revisão de práticas pedagógicas adotadas nas IES para esse fim. Nesse seguimento, a equipa do projeto, estruturou e dinamizou um curso de educação para o Pensamento Crítico para professores de IES europeias (Dominguez, 2018b), cuja primeira edição ocorreu em 2018, em Roma, Itália. Os autores deste trabalho frequentaram o curso mencionado e participaram na sua replicação em Vila Real, Portugal. O curso foi estruturado em sessões plenárias e atividades práticas de trabalho em grupo segundo três grandes tópicos de formação: planificação, métodos de ensino e de aprendizagem, e avaliação do PC. Como formandos e formadores destes cursos, integrámos na prática letiva os elementos associados ao PC ao nível das unidades curriculares de acordo com as orientações preliminares para a qualidade da educação do PC de Dominguez (2018b, p. 56), Figura 1.

1. Definir os objetivos do curso com uma descrição explícita dos objetivos e resultados de aprendizagem esperados em termos de pensamento crítico;

2. Avaliar as necessidades de pensamento crítico dos alunos usando diferentes métodos, como diagnóstico (de acordo com o ano do curso e antecedentes e necessidades do mercado de trabalho);

3. Alinhar os objetivos do curso de pensamento crítico com os objetivos da unidade curricular;

4. Projetar um conjunto de atividades de aprendizagem envolventes que levem a os resultados de aprendizagem estabelecidos para o pensamento crítico;

5. Fornecer às atividades de aprendizagem do pensamento crítico oportunidade de transferir as diferentes competências ou disposições em várias e diferentes situações;

6. Fornecer recursos de aprendizagem do pensamento crítico relacionados com as necessidades dos alunos, futuros profissionais;

7. Promover a autorregulação dos alunos através de atividades de aprendizagem pensamento crítico, avaliação formativa e oportunidades de autoavaliação:

Figura 1 - Orientações preliminares ao nível das UC 
Neste sentido, narram-se três estudos de caso que tiveram como mote a promoção do PC em três unidades curriculares (UC) da área de matemática de diferentes licenciaturas de uma IES do norte de Portugal. Apresentam-se as abordagens utilizadas nestas UC enquadrando o PC nas práticas letivas e as modificações concretizadas ao nível da planificação, estratégias adotadas e avaliação das aprendizagens.

\section{Metodologia}

A presente investigação é um trabalho de natureza qualitativa e descritiva (Cohen, Manion \& Morrison, 2011). As intervenções relatadas abordam os seguintes aspetos: a planificação usando o modelo de desenho instrucional 4C/ID (van Merriënboer, Clark \& Croock, 2002), os métodos de ensino e aprendizagem promotores de PC, e a avaliação do PC. No modelo de desenho instrucional adotado há quatro componentes inter-relacionados essenciais para a aprendizagem complexa: tarefas de aprendizagem; informação de apoio; informação dada no momento, just-in-time; e tarefas. Os métodos instrucionais de cada componente são agrupados aos processos de aprendizagem (van Merriënboer, Clark \& Croock, 2002).

Participaram no estudo 179 alunos de uma IES pública do norte de Portugal distribuídos por 3 UC de diferentes licenciaturas que decorreram no ano letivo de 2017/18 (Figura 2).

\begin{tabular}{|c|c|c|c|c|c|}
\hline Caso & $\begin{array}{c}\text { Licenciatura } \\
\left(1 .^{\circ} \text { ciclo }\right)\end{array}$ & $\begin{array}{l}\text { Unidade curicular } \\
\text { (ano curicular/ } \\
\text { semestre) }\end{array}$ & Área & Professor & $\begin{array}{l}\mathrm{N} .^{\circ} \text { de } \\
\text { alumos }\end{array}$ \\
\hline Caso 1 & $\begin{array}{l}\text { Comunicação } \\
\text { e Multimédia }\end{array}$ & $\begin{array}{c}\text { Álgebra linear } \\
1 . \% 2 .^{\circ}\end{array}$ & $\begin{array}{l}\text { Álgebra } \\
\text { linear }\end{array}$ & $\begin{array}{c}\text { Professores } \\
\text { A e B }\end{array}$ & 79 \\
\hline Caso 2 & Biologia & $\begin{array}{c}\text { Bioestatística e } \\
\text { delineamento } \\
\text { experimental } \\
1 . \% 2 .^{\circ} \\
\end{array}$ & Estatística & $\begin{array}{c}\text { Professora } \\
\text { C }\end{array}$ & 78 \\
\hline Caso 3 & Serviço Social & $\begin{array}{c}\text { Metodologias de } \\
\text { investigação social } \\
\text { aplicadas ao serviço } \\
\text { social II } \\
2 . \% 2 .^{\circ}\end{array}$ & Estatística & $\begin{array}{c}\text { Professora } \\
\text { D }\end{array}$ & 29 \\
\hline
\end{tabular}

Figura 2 - Resumo dos participantes de cada caso

Os aspetos do curso de formação em PC integrados na prática letiva são apresentados na descrição de cada caso.

\section{Caso 1 - Álgebra linear}

Os participantes do Caso 1 - Álgebra linear eram alunos da UC de Álgebra Linear $(\mathrm{AL})$, do $2 .^{\circ}$ semestre do $1 .^{\circ}$ ano do $1 .^{\circ}$ ciclo em Comunicação e Multimédia (CM). A 
UC funcionou com uma turma única para as aulas teóricas (T), com o total de 79 alunos inscritos em 3 turmas de aulas prática laboratorial (PL): 26 alunos na turma PL1, 26 alunos na PL2 e 27 na PL3. A UC de AL teve dois professores integrados na sua lecionação, sendo o professor A responsável pela turma T (2h/semana) e por uma das turmas PL, o professor B foi responsável pelas restantes turmas PL (2h/semana). Do total de alunos inscritos em AL, cerca de $42 \%$ tinham pelo menos duas reprovações nesta UC.

Na Figura 3 apresenta-se a planificação geral desta UC usando o modelo 4C/ID (van Merriënboer et al., 2002). Foi definido um objetivo de aprendizagem a alcançar pelos alunos no final do semestre que foi, então, particionado em cinco etapas e cinco momentos de avaliação.

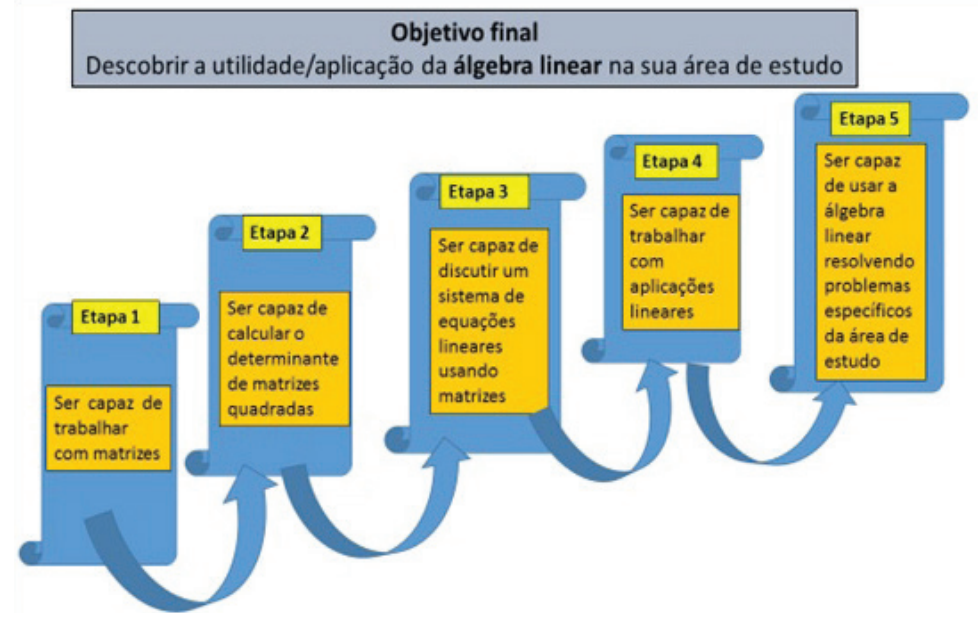

Figura 3 - Modelo 4C/ID usado no planeamento de AL

A licenciatura de CM é vocacionada para o uso de imagens, vídeos e outros tipos de media. Tendo este aspeto em mente, os professores desta UC desafiaram-se a si próprios para também usarem imagens como recurso base de suporte aos processos de ensino e de aprendizagem. As imagens, quando usadas no ensino, promovem uma melhoria na aprendizagem (Maciel, Rêgo \& Carlos, 2017).

Neste sentido, em duas tarefas principais foram utilizadas imagens sobre os conteúdos programáticos. Este uso de imagens foi a inovação em relação a todos os outros anos letivos anteriores. A primeira tarefa consistiu na procura de uma definição adequada de "matriz". Foram usadas várias imagens como as apresentadas na Figura 4 (à esquerda), e pedido aos alunos que as observassem de forma a retirarem aquilo que entendessem serem dados importantes para definir apropriadamente o conceito de "matriz". Uma outra tarefa, igualmente com recurso a imagens, que tinha como 
objetivo "Identificar e investigar sobre as operações algébricas com matrizes" (Figura 4, à direita). Neste caso, optou-se por apresentar as referidas imagens, levando o aluno a observar, questionar, argumentar e descobrir por si próprio as operações aí apresentadas e possíveis com as matrizes.
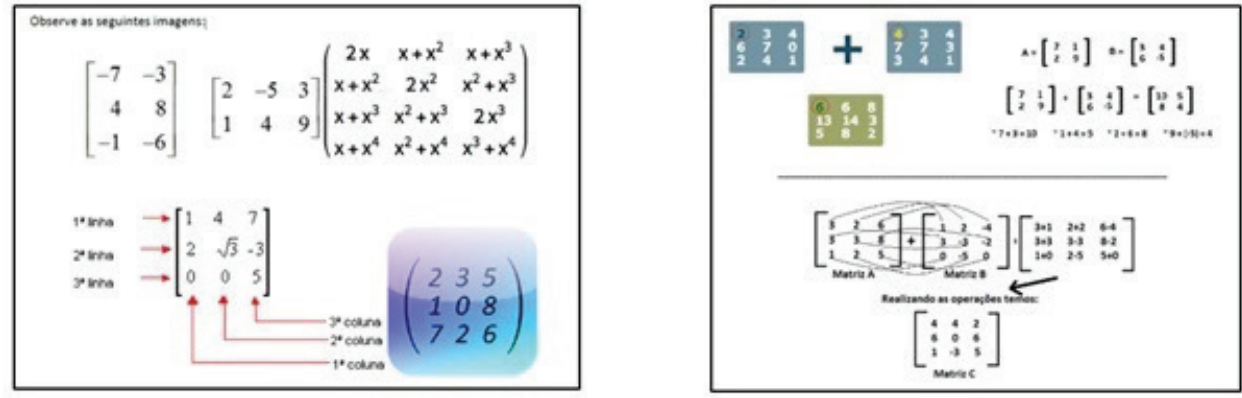

Figura 4 - Excertos de imagens usados nas aulas PL de AL

Cada etapa preconizada no planeamento geral da UC (Figura 2) foi abordada, de uma forma geral e sistemática, através de um conjunto de tarefas propostas com recurso a imagens sobre os diferentes conteúdos programáticos de AL. As tarefas foram propostas nas aulas $\mathrm{PL}$, onde a observação das imagens tinha como consequência a reflexão e o questionamento, primeiro de forma individual e, depois, em grande grupo na turma. O professor da turma tinha um papel de mediador das reflexões e questões que iam surgindo ao longo da aula. $\mathrm{Na}$ aula $\mathrm{T}$ seguinte os alunos eram relembrados sobre os conteúdos abordados na atividade da aula PL anterior e, no final da aula, os alunos ficavam com o registo escrito dos conceitos e exemplos mais importantes.

Foi proposta uma avaliação periódica com cinco momentos de avaliação ao longo do semestre, promovendo o "feedback" contínuo entre professores e alunos: dúvidas e respostas do(a) professor(a). Os quatro primeiros momentos de avaliação incidiram em conceitos básicos e essenciais para as etapas seguintes, enquanto o último focou aspetos mais globais onde já se articulavam todos os conteúdos programáticos.

Dos vários tipos de avaliação que promovem o desenvolvimento do PC no aluno, um deles é o teste de escolha múltipla (Sugrue, 1995), que permite avaliar o valor lógico de afirmações que são apresentadas e relacionadas com um determinado conteúdo. O aluno, perante esta avaliação, terá de refletir e argumentar para fundamentar o seu juízo do valor lógico que atribui à afirmação. Em termos de PC, o aluno tem de apelar às suas competências de interpretação, análise e de explicação. Nas avaliações escritas, com o questionamento adotado, tentou-se que os alunos aplicassem as suas competências de PC de interpretação, análise e de explicação. Na Figura 5 apresenta-se um exemplo de um conjunto destas afirmações para as quais os alunos devem responder verdadeiro ou falso e justificar com exemplos. 
Indique, justificando convenientemente a sua resposta, o valor lógico das seguintes afirmações (V-verdadeiro ou F-falso):

a) Toda a matriz triangular superior está na forma de escada de linhas.

b) Para duas quaisquer matrizes quadradas $\mathrm{A}$ e $\mathrm{B}$ da mesma ordem, verifica-se sempre que $(\mathrm{A}+\mathrm{B})^{2}=\mathrm{A}^{2}+2 \mathrm{AB}+\mathrm{B}^{2}$.

c) Um sistema com 3 equações e 2 incógnitas é sempre possível indeterminado.

d) Sejam A e B duas matrizes quadradas da mesma ordem com as entradas todas diferentes de zero. Então $\mathrm{AB}$ é sempre diferente da matriz nula.

e) Sejam $\mathrm{A}$ e B matrizes quaisquer quadradas da mesma ordem. Então $|\mathrm{AB}|=|\mathrm{BA}|$.

f) $\operatorname{car}(\mathrm{A}+\mathrm{B})$ é sempre diferente de $\operatorname{car}(\mathrm{A})+\operatorname{car}(\mathrm{B})$, onde $\operatorname{car}(\mathrm{X})$ representa a característica da matriz X.

g) O vetor que tem coordenadas $(1,2)$ na base $((1,2),(2,3))$ é o vetor $(5,8)$.

h) É sempre possível multiplicar uma matriz linha por uma matriz coluna.

i) Sejam u, v e w vetores linearmente dependentes. Então u é combinação linear de v e w.

j) Seja $\mathrm{C}$ uma matriz com $\mathrm{n}$ linhas e $\mathrm{n}$ colunas então $|-\mathrm{C}|=|\mathrm{C}|$ para qualquer $\mathrm{n} .{ }^{\circ}$ natural $\mathrm{n}$.

Figura 5 - Propostas num momento de avaliação escrito na UC de AL, questões verdadeiro/falso

\section{Caso 2 - Bioestatística e delineamento experimental}

O Caso 2 - Bioestatística e delineamento experimental (BDE) relata a experiência com alunos do $1 .^{\circ}$ ciclo em Biologia que frequentavam esta UC no ano letivo 2017/18.

Os principais objetivos de BDE visam a apresentação, com algum rigor matemático, dos principais métodos da estatística e a aplicação desses métodos às ciências biológicas. Os conteúdos programáticos estão divididos em duas partes principais teoria das probabilidades e inferência estatística - intercaladas por uma breve abordagem aos conceitos e técnicas básicas de estatística descritiva. A professora $\mathrm{C}$ teve o propósito de selecionar estratégias de envolvimento ativo dos alunos (O'Connor, 2013) que os motivem a adquirir os conhecimentos, e a transferir esses conhecimentos para a resolução de problemas em situações aplicadas ao mundo real, em particular na Biologia. Usou-se o modelo de planificação 4C/ID (van Merriënboer et al., 2002) para atingir esses objetivos. Na implementação, delineou-se uma sequência de tarefas organizadas em fases de conhecimentos integrados para cada parte do programa de BDE. A Figura 6 apresenta o esquema concebido para cada um dos cenários da teoria das probabilidades de BDE. 


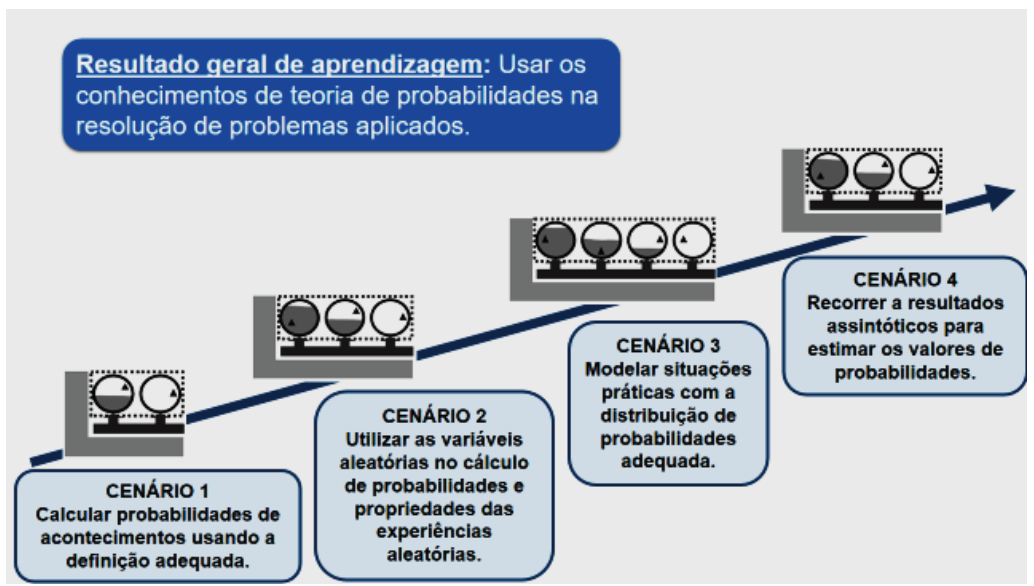

Figura 6 - Modelo 4C/ID usado no planeamento de BDE

Apesar de ser uma UC de formação base dos alunos lecionada no $2 .^{\circ}$ semestre do $1 .^{\circ}$ ano curricular da licenciatura, dos 76 alunos inscritos, 31 alunos (41\%) tinham mais de uma inscrição encontrando-se a frequentar UC dos $2 .^{\circ}$ e $3 .^{\circ}$ anos curriculares.

Quanto às modificações, a docente abandonou a metodologia nas aulas $T$ utilizada no ano letivo anterior, em que a transmissão dos conhecimentos era feita através da exposição com projetor multimédia acompanhada da apresentação de exemplos, ocasionalmente com a utilização do quadro branco. Como novidade nesta UC, no final de 11 das aulas $T$ lecionadas os alunos realizaram individual ou cooperativamente uma atividade de avaliação sobre os conteúdos abordados na aula, através do preenchimento de um questionário (Q) na plataforma Google Forms no portátil ou telemóvel. O $Q$ foi adotado como uma técnica de avaliação formativa (Lopes \& Silva, 2012), com o objetivo de recolher informações e perceber se os alunos entenderam os conteúdos programáticos dessa aula. Nestes $Q$ as questões eram fechadas, de escolha múltipla, do tipo Verdadeiro/Falso ou questões com resposta numérica. A maioria das questões requeria a reflexão individual e/ou a troca de ideias com colegas para a tomada de uma decisão quanto à resposta correta. Nas questões do tipo Verdadeiro/Falso (Sugrue, 1995), o aluno procura justificações face à afirmação proposta e tem de recorrer às suas competências de PC de interpretação, análise e explicação. O tempo necessário para a resposta a cada $Q$ variou de 10 a 20 minutos, os alunos tomavam conhecimento imediato do resultado no final do preenchimento e, após a aula, era disponibilizada uma folha de soluções. O feedback era dado não só com o objetivo de os alunos identificarem as falhas cometidas e melhorarem o seu desempenho no futuro, mas também para auxiliar o professor no desenho e preparação das estratégias de aprendizagem nas aulas seguintes (Collis, De Boer \& Slotman, 2001). Como os resultados 
nos $Q$ tinham o peso de $10 \%$ na nota final, aumentou o número médio de alunos que frequentavam as aulas T. Na Figura 7 apresenta-se um exemplo de uma pergunta de um desses questionários.

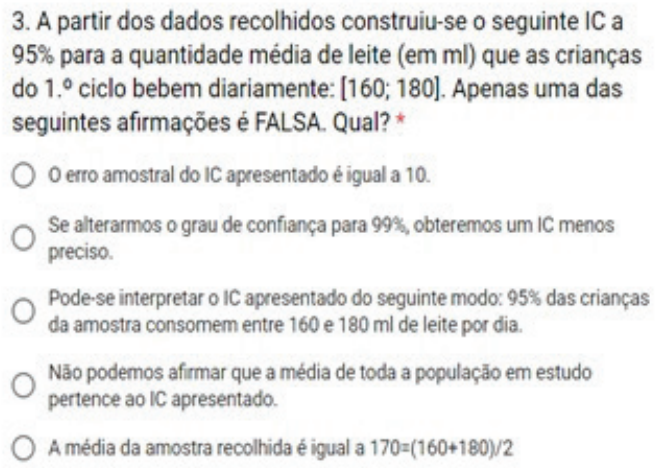

Figura 7 - Exemplo de pergunta colocada no questionário de avaliação formativa da UC de BDE

Nas aulas TP privilegiou-se o trabalho cooperativo (trabalho expositivo anteriormente feito na totalidade pela professora) para a resolução de problemas aplicados aos conceitos já abordados nas aulas T, usando o método cooperativo "Pensar Formar Pares - Partilhar" (Lopes \& Silva, 2009). Nas aulas T também se recorreu ao mesmo método cooperativo na abordagem de determinados conteúdos. A nova metodologia consistiu na introdução dos vários conceitos e resultados do programa com exemplos da sua aplicação - Pensar - e utilização - Formar Pares - em quase todas as aulas, discutidos depois em grande grupo - Partilhar. Os principais objetivos da metodologia incluem a "partilha de informação, escuta ativa, discussão de ideias, reforço e aprofundamento da aprendizagem, desenvolver a criatividade, o pensamento crítico e a autoestima" (Lopes \& Silva, 2009, p. 142), o que foi conseguido nas aulas T e TP deste ano letivo.

\section{Caso 3 - Metodologias de Investigação Social Aplicadas ao Serviço Social II (MISASS II)}

A descrição relativa ao Caso 3 - MISASS II, relata a experiência com alunos da licenciatura em Serviço Social numa das turmas TP de MISASS II. Os objetivos desta UC consistem no conhecimento e aplicação dos principais métodos da estatística em serviço social. Os conteúdos programáticos estão divididos em duas partes: estatística descritiva e inferência estatística. A professora $D$ selecionou estratégias de aprendizagem para envolver os alunos na aprendizagem e na resolução de problemas em casos concretos de serviço social. Na Figura 8, apresenta-se o modelo 4C/ID (van 
Merriënboer et al., 2002) com a planificação geral de MISASS II. Foi definido um objetivo geral a alcançar pelos alunos e esse objetivo foi subdividido em 4 etapas (settings).

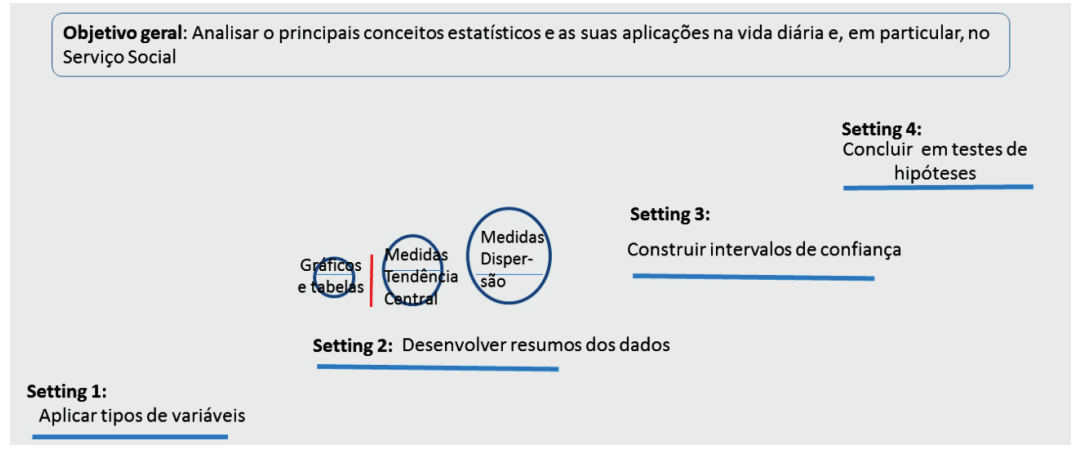

Figura 8 - Modelo 4C/ID em MISASS II

A UC de MISASS II é lecionada no $2 .^{\circ}$ semestre do $2 .^{\circ}$ ano curricular da licenciatura e apenas cerca de metade dos alunos inscritos na UC (29 alunos, 48\%) frequentavam a turma TP a cargo da professora D em 2017/2018. Dos 29 alunos da turma, apenas 4 (14\%) eram rapazes e apenas 8 dos alunos (28\%) tinham mais de uma inscrição. Esta UC dispõe de dois blocos TP de 2 horas/semana. Nesta implementação, abandonou-se a exposição com projetor multimédia de exemplos e uso do quadro branco. Antes das aulas eram disponibilizados aos alunos os materiais escritos (resumos, exemplos e problemas) e eles traziam dúvidas para as aulas sobre os tópicos em estudo. Nas aulas privilegiou-se o trabalho cooperativo de pares ou trios de alunos na resolução dessas tarefas. Como os alunos deveriam ter estudado os materiais teóricos - "Pensar" -, chegavam à aula e pensavam em pares ou trios - Formar Pares - e partilhavam os resultados em grande grupo para se poder fazer um ponto da situação - Partilhar: método cooperativo "Pensar - Formar Pares - Partilhar" (Lopes \& Silva, 2009). O feedback era dado, com o objetivo de os alunos identificarem as falhas cometidas, melhorarem o seu desempenho e motivá-los (Silva \& Lopes, 2015).

À semelhança de anos letivos anteriores, ao longo do semestre dedicaram-se aulas à realização de um trabalho de projeto $(P)$ em grupos com 2 ou 3 alunos. A forma como a professora escolheu os grupos foi diferente da anterior (numerando os alunos e sorteando os elementos do grupo). A aprendizagem baseada em projetos é importante no ensino de estatística (Koparan \& Güven, 2015) pois os alunos integram todos os aspetos de formulação do problema à sua resolução, passando pela recolha e análise de dados estatísticos. Nos $\mathrm{P}$ o trabalho dos grupos foi faseado e o produto final foi um poster, incluindo a apresentação oral de cada grupo. O P desenvolveria as competências de interpretação, explicação, análise e inferência e as disposições de sistematicidade, mente analítica e, na apresentação oral, a autoconfiança. Os alunos 
também analisaram um artigo, mas por falta de tempo os alunos só efetuaram a parte do autor. A análise do artigo pedida aos alunos foi feita com base na grelha FRISCO de Ennis (Dominguez et al., 2015). A grelha FRISCO permitiu aos alunos desenvolver algumas competências de pensamento crítico: identificação de razões, inferências, credibilidade das informações, entre outras. A grelha foi debatida na aula através de um exemplo da análise de um artigo de jornal e, após o debate, os alunos fizeram a análise de outro artigo.

\section{Análise dos resultados}

\section{Caso 1 - Álgebra Linear}

Quanto à implementação de tarefas com o uso de imagens, e como resultado positivo, apraz-nos verificar um aumento da assiduidade dos alunos comparativamente com todos os anos letivos anteriores. Na Figura 9 apresentamos taxas relativas a 2016/17 e 2017/18, o ano desta implementação. As estatísticas de assiduidade (a taxa de Avaliados/admitidos passou de $77.8 \%$ para 95.6\% de 2016/17 para 2017/18, Figura 9) e de aproveitamento escolar - Avaliados/admitidos, Aprovados/inscritos e Aprovados/Admitidos foram as que apresentaram maiores incrementos em 2017/18.

$\begin{array}{cl}\text { Ano letivo de } & \mathbf{2 0 1} \\ \text { Alunos: } \\ \text { Inscritos: } & 89 \\ \text { Admitidos: } & 72 \\ \text { Avaliados: } & 56 \\ \text { Aprovados: } & 47 \\ \text { Reprovados: } & 9\end{array}$

Rácios:

Admitidos/Inscritos: $\quad 80.9 \%$ Avaliados/Inscritos: $\quad 62.9 \%$ Avaliados/Admitidos: Aprovados/Inscritos: Aprovados/Admitidos: Aprovados/Avaliados: Reprovados/Inscritos: Reprovados/Admitidos: Reprovados/Avaliados:

\section{Ano letivo de 2017/2018 \\ Alunos:}

Inscritos: 82

Admitidos: 72

Avaliados: 69

Aprovados: 60

Reprovados: 9

Figura 9 - Estatísticas de aproveitamento de dois anos letivos 
Pensamos que estes resultados se ficaram a dever ao uso das imagens nas tarefas das aulas que despertou a curiosidade dos alunos, proporcionou o debate e potenciou que encontrassem soluções e conclusões através de critérios relevantes (Maciel et al., 2017). O aluno passou a ter um papel mais ativo na sua aprendizagem, participando com entusiamo nas atividades letivas: trabalharam em pares ou trios e junto do(a) professor(a) da turma solicitavam apoio. Esta estratégia potenciou as competências de interpretação, análise, explicação e autorregulação e as disposições de autoconfiança e curiosidade intelectual.

A análise dos resultados aqui apresentados reporta-se à deteção de erros nos testes, pois a análise de conteúdo das respostas dos alunos nos testes realiza-se com base no respetivo material escrito (Cohen et al., 2011). As categorias que emergiram foram as dos erros dos alunos às respostas avaliadas pelos professores com mais de 15 anos de experiência nesta área do ensino superior.

Num dos testes de AL de 2017/18 foram pedidos aos alunos exemplos, caso fosse possível, de matrizes com as propriedades estudadas. Esta questão foi colocada de uma forma oposta ao usual que era, por exemplo, a de verificar se duas matrizes dadas satisfazem certa propriedade ou igualdade. Neste (novo) questionamento, para além de usar conceitos de AL, o aluno reflete sobre qual será o exemplo a usar, isto é, aplica as suas competências de PC de interpretação, análise e de explicação. Como situação de erro na resposta dada à questão 4.d) apresenta-se um exemplo na Figura 10.

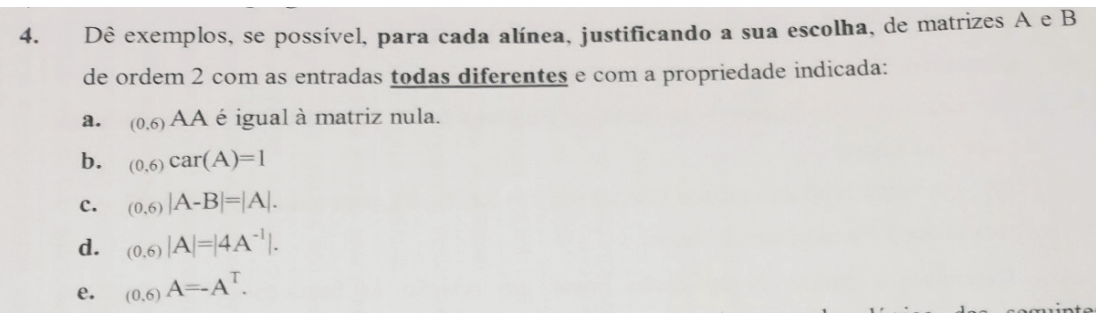

Figura 10 - Exemplo de questões, testes AL 2017/18

Num exemplo, um aluno cometeu vários erros de cálculo: identificou a inversa de uma matriz invertível A como sendo um número, o inverso do determinante dessa matriz A; e, além disso, não sabia que é impossível existir divisão por zero. Mais ainda, identificou a divisão por zero como sendo igual a zero.

Noutro exemplo de questões usadas nos testes da UC de AL 2017/18, os alunos devem apresentar o valor lógico de cada afirmação, bem como a respetiva justificação. Nas justificações, os alunos, para além de terem de dominar conceitos de AL, têm de saber a razão pela qual atribuíram o valor lógico, isto é, aplicar as suas competências de PC de interpretação, análise e de explicação (Figura 11). 
5. Indique, justificando convenientemente a sua resposta, o valor lógico das seguintes afirmações (V-verdadeiro ou F-falso):

a. $(0,5)$ Toda a matriz de ordem 2 triangular superior está em escada de linhas.

b. $(0.5)$ É sempre possivel multiplicar uma matriz coluna por uma matriz linha.

c. $(0,5) \mathrm{A}$ caracteristica da matriz simples pode ser superior à característica da matriz ampliada.

d. $\quad(0,5)\left|A\left(A^{-1} B\right)^{T}\left(B A^{T}\right)^{-1}\right|=|A|$.

e. $(0,5) \operatorname{car}(\mathrm{A}+\mathrm{B})$ pode ser igual a um mesmo que car $(\mathrm{A})$ e $\operatorname{car}(\mathrm{B})$ sejam diferentes de um, onde $\operatorname{car}(\mathrm{X})$ representa a característica da matriz $\mathrm{X}$

f. $(0,5)$ Se os vetores $u, v$ e $w$ são linearmente independentes também os vetores $3 u-v+w$, $w+2 u, v+u$ são linearmente independentes.

g. (0.5) Se $A$ e $B$ são matrizes invertiveis da mesma ordem então $A B$ é ainda invertivel $(A B)^{-1}=B^{-1} A^{-1}$

Figura 11 - Exemplo de questões, testes AL 2017/18

Nas respostas dadas à questão 5.g) da Figura 11, também se encontrou outro erro: alguns alunos não identificam qualquer diferença entre uma matriz e um número, efetuando com matrizes os mesmos procedimentos que com números.

Apesar de as questões serem mais exigentes e terem conduzido ao mesmo tipo de erros que tinham surgido em anos letivos anteriores, os alunos foram mais assíduos às aulas $T$ e PL pois o uso das imagens na aula $T$ e das tarefas com imagens nas aulas $P L$ foram desafiantes.

\section{Caso 2 - Bioestatística e delineamento experimental}

$\mathrm{Na}$ análise dos resultados da BDE foram avaliadas as notas dos $\mathrm{Q}$ implementados, individual ou cooperativamente, bem como no questionário de opinião através do "Google Forms". Este último Q foi solicitado aos alunos no final do semestre e visava recolher as opiniões dos alunos, quer sobre as aulas, quer sobre os elementos do pensamento crítico introduzidos.

Os questionários das aulas T levaram ao aumento do número médio de alunos que frequentavam as aulas T, de 20 alunos por aula no ano letivo 2016/17 (29\% dos 68 alunos, turma T) para 28 alunos por aula no ano letivo 2017/18 (39\% dos 71 alunos, turma T). Nos resultados médios por Q (como se constata na Figura 12, 11 questionários realizados), não houve uma tendência geral de evolução positiva com exceção dos últimos três $Q$. O resultado médio mais baixo corresponde ao Q08 em que era feita a primeira avaliação sobre inferência estatística. Este resultado pode estar associado às dificuldades de abstração matemática e raciocínio estatístico que conduzem a frequentes dificuldades na compreensão de conceitos de inferência estatística e na utilização dos métodos estatísticos (Garfield \& Ben-Zvi, 2007). Estes questionários foram introduzidos pela primeira vez na UC BDE e permitiram 
a autorregulação da aprendizagem dos próprios alunos (que podiam ver logo os resultados obtidos e podiam efetuar a sua correção), bem como aumentar a sua autoconfiança.

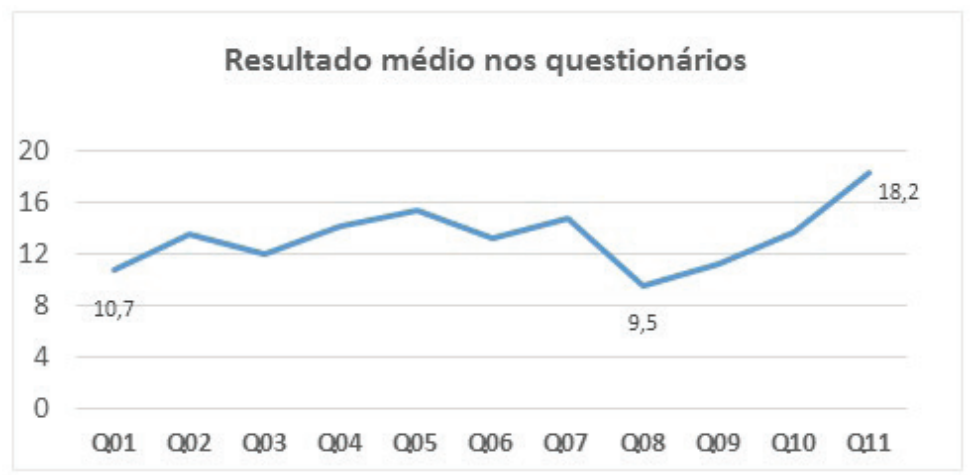

Figura 12 - Resultado médio dos alunos nos Q da BDE

No preenchimento do $Q$ de opinião dos alunos de BDE, apenas 13 alunos responderam (17\% dos 76 alunos), com dois alunos a responderem que não frequentaram as aulas $T$ pelo que não se consideraram as suas respostas. Sobre a questão "Se esta unidade curricular decorrer novamente no próximo ano letivo: quais os elementos (relacionados com o desenvolvimento do PC) que devem ser mantidos? Porquê?", 73\% (8) dos alunos consideraram que todos os elementos de avaliação deveriam ser mantidos e 18\% (2) referiram especificamente os questionários feitos nas aulas T. Destaca-se a resposta dada pelo aluno 4: "acho que o método de avaliação foi bem delineado (...) deu aos alunos uma grande margem de manobra para que se pudessem aplicar e perceber toda a matéria". Já o aluno 7, que menciona também o papel do professor no desenvolvimento dos $Q$ durante o curso: "A própria existência do questionário. É interessante (...) como também para quem o está a fazer, ver as diferenças no pensamento crítico e o seu desenvo/vimento." Quanto à questão "Se esta unidade curricular decorrer novamente no próximo ano letivo: quais os elementos (relacionados com o desenvolvimento do PC) que devem ser alterados? Porquê?", nenhum aluno apontou alterações aos elementos desta UC e o aluno 9 mencionou: "Nenhum pois todos foram devidamente incutidos e fizeram com que o desenvolvimento do nosso PC aumentasse." Na opinião da professora C, os testemunhos dos alunos no $Q$ de opinião denotam uma consciência do seu desenvolvimento enquanto pensadores críticos.

\section{Caso 3 - MISASS II}

Em MISASS II foram analisadas as respostas no $Q$ opinião sobre a UC solicitado aos alunos no final do semestre, a que os 22 alunos (76\%) da turma presentes na última aula responderam. 59\% dos alunos afirmaram que frequentaram com regularidade 
as aulas e 54\% estão de acordo com a afirmação "Gostei de frequentar esta UC no 2. semestre de 2017/18". Sobre a forma como a professora escolheu os grupos, dos 22 alunos apenas um, aluno 1, não esteve de acordo: "Mudaria a escolha dos elementos do grupo de forma aleatória pelo programa uma vez que não sendo da mesma zona geográfica dificultou um pouco esta tarefa."

Nas aulas, a professora D verificou de forma positiva o envolvimento ativo dos alunos nas tarefas. Foi notória a evolução dos alunos sobretudo nas competências de interpretação, explicação, análise e nas disposições de sistematicidade, mente analítica e de autoconfiança. Já no $\mathrm{P}$ os alunos foram relatando que estavam a gostar de trabalhar com pessoas diferentes e estavam a conseguir resolver os problemas de funcionamento dos grupos. Além das mencionadas, evidenciaram-se competências de inferência e de autorregulação: os alunos conseguiram ir discutindo com a professora os seus resultados e as apresentações foram bem preparadas. Quanto às disposições, evidenciaram-se a curiosidade intelectual e a autoconfiança através das observações da professora D. Além disso, $82 \%$ de alunos estiveram de acordo com o facto de o trabalho de grupo ter resultado e 14 alunos (64\%) dos alunos mencionaram o $\mathrm{P}$ como o que mais gostaram na UC. Dos 22 alunos, 73\% gostaram da estruturação do $\mathrm{P}$ por etapas e com a apresentação final de um poster. Entre as suas razões destacam-se as do aluno 2: "Não mudaria porque considero que a elaboração do TP por fases é muito benéfico pois quando temos que apresentar o $P$ final apenas é necessário juntar todos os elementos antes elaborados." No próximo ano letivo $73 \%$ manteriam o trabalho nos moldes realizados, 14\% escreveram "não sei" e os restantes 14\% desejariam "A realização de aulas com a matéria explicada" e "começar a fazer exercícios sem a explicação da professora". No entanto, o aluno 3 referiu: "Correção de todas as fichas uma vez que assim ajudaria meIhor para a preparação da frequência, apesar de a professora se mostrar sempre disponível para ajudar no esclarecimento de dúvidas no período de aulas e extra aulas." Entre as razões da manutenção do trabalho citam-se as do aluno 4: "Considero que as fichas de exercícios existentes no SIDE são uma mais-valia no aprofundamento dos conteúdos teóricos da UC (...) para nos ajudar (...) fora das aulas. Serviram como fio condutor sobre tudo o que foi lecionado." Também houve quem concordasse, mas... como o aluno 6: "Considero que as fichas de exercícios existentes no SIDE são uma mais-valia no aprofundamento dos conteúdos teóricos da UC. Por vezes tornava-se cansativo conciliar as várias fases do P com os trabalhos das outras UC."

\section{Reflexão final}

Enquanto docentes do ensino superior na área da Matemática, preocupados em promover o PC dos nossos alunos e futuros profissionais no mais variados domínios do conhecimento, destacamos a nossa ligação e participação no projeto CRITHINKEDU. 
A formação adquirida no âmbito deste projeto incutiu-nos uma preocupação mais acentuada relativamente a aspetos fundamentais no ensino do PC. A frequência do curso de Roma (Dominguez, 2018b), e a sua replicação em Portugal, revelou-se uma aprendizagem e um desafio constantes enquanto elementos de uma comunidade de prática europeia sobre PC no ensino superior.

Nos três casos relatados é patente a influência do curso de Roma na preparação mais sistematizada e organizada das UC (Dominguez, 2018b). O facto de termos contactado com o modelo 4C/ID (van Merriënboer et al., 2002) foi uma mais-valia no repensar das práticas letivas (figuras 2, 5 e 7) e na sua sistematização. Todas as intervenções realizadas tiveram subjacente um trabalho ativo dos alunos, envolvendo-os nas aulas. Por exemplo, usando imagens (Maciel et al., 2017), questionários (Sugrue, 1995) e trabalho de projeto (Koparan \& Güven, 2015). Nos três casos, foram usados vários aspetos do feedback (Silva \& Lopes, 2015).

Para além de alinhar os objetivos das UC com os do PC e apesar das modificações introduzidas, ainda falta incluir os elementos da avaliação do PC dos alunos (pré e pós intervenção, ponto 2 da Figura 1). Em intervenções futuras, as atividades a desenvolver continuam a ter que ser orientadas para obter resultados efetivos em termos do PC para os alunos e, portanto, desenvolver a transferência das competências adquiridas nestas UC para a sua profissão (Dominguez, 2018a), nomeadamente em relação aos pontos 6 e 7 das orientações preliminares citados na Figura 1.

Na perspetiva dos professores, o peso dos (novos) elementos na nota final na UC terá levado ao aumento da frequência às aulas e à natural adesão dos alunos às novas atividades. Deste envolvimento, em nossa opinião, resultou um maior desenvolvimento do PC, manifestado pelas menções ao PC pelos alunos no caso 2 - BDE.

A partir do relato dos três casos fica para trabalho futuro a análise detalhada dos documentos recolhidos (testes, relatórios, posters do trabalho de projeto). Temos como propósito continuar a implementar melhorias nas nossas práticas letivas e manter-nos ligados à comunidade de prática que se alargou com o projeto CRITHINKEDU.

\section{Referências Bibliográficas}

Collis, B., De Boer, W., \& Slotman, K. (2001). Feedback for web-based assignments. J. of Computer Assisted Learning, 17(3), 306-313.

Cohen, L., Mannion, L., \& Morrison, K. (2011). Research Methods in Education (7th Ed). New York: Routledge.

Cruz, G., Nascimento, M., Payan, R., Dominguez, C., Silva, H., \& Morais, F. (2017). From labor market needs to critical thinking across the European High Education Curricula. EAIR 39th Annual Forum, Porto, Portugal. 
Dominguez et al., (2015). Adding value to the learning process by online peer review activities: towards the elaboration of a methodology to promote critical thinking in future engineers. European J. of Engineering Ed., 40(5), 573-591.

Dominguez, C. (coord.) (2018a). A European collection of the Critical Thinking skills and dispositions needed in different professional fields for the 21st century. Vila Real: UTAD. ISBN: 978-989-704256-0.

Dominguez, C. (coord.) (2018b). The CRITHINKEDU European course on critical thinking education for university teachers: from conception to delivery. Vila Real: UTAD. ISBN: 978-989-704-274-4.

Dominguez, C. (coord.) (2018c). A European review on Critical Thinking educational practices in Higher Education Institutions. Vila Real: UTAD. ISBN: 978-989-704-258-4.

Facione, P. (1990). Critical Thinking: A Statement of Expert Consensus for Purposes of Educational Assessment \& Instruction: The Delphi Report. California: California Academic Press.

Garfield, J., \& Ben-Zvi, D. (2007). How students learn statistics revisited: A current review of research on teaching and learning statistics. International Statistical Review, 75(3), 372-396.

Huang, H., Ricci, F., \& Mnatsakanian, M. (2016). Mathematical teaching strategies: Pathways to critical thinking and metacognition. International J. of Research in Ed. and Science, 2(1), 190-200.

Jacob, S. (2012). Mathematical achievement and critical thinking skills in asynchronous discussion forums. Procedia-Social and Behavioral Sciences, 31, 800-804.

Koparan, T., \& Güven, B. (2015) The effect of project-based learning on students' statistical literacy levels for data representation, International J. of Mathematical Ed. in Science and Technology, 46(5), 658-686.

Lopes, J., \& Silva, H. (2009). Aprendizagem cooperativa na sala de aula: um guia prático para o professor. Lisboa: Edições Lidel.

Lopes, J., \& Silva, H. (2012). 50 Técnicas de avaliação formativa. Lisboa: Ed. Lidel.

Maciel, A., Rêgo, R., \& Carlos, E. (2017). Possibilidades pedagógicas do uso da Imagem Fotográfica no Livro Didático de Matemática, Bolema, 31(57), 344-364.

O'Connor, K. (2013). Class participation: Promoting in-class student engagement. Education, 133(3), 340-344.

Silva, H., \& Lopes, J. (2015). Eu, professor, pergunto: 20 respostas sobre planificação do ensinoaprendizagem, estratégias de ensino e avaliação. Lisboa: Ed. Lidel.

Silva, H., Lopes, J., Dominguez, C., Payan-Carreira, R., Morais, E., Nascimento, M., \& Morais, F. (2016). Fostering critical thinking through peer review between cooperative learning groups. Revista Lusófona de Educação, 32, 31-45. [Disponível em http://revistas.ulusofona.pt/index.php/rleducacao/article/ view/5511, consultado 3 de Julho de 2018].

Sugrue, B. (1995). A theory-based framework for assessing domain-specific problem-solving ability. Ed. Measurement: Issues and Practices, 14(3), 29-36.

van Merriënboer, J., Clark, R., \& Croock, M. de. (2002). Blueprints for Complex Learning: The 4C/IDModel, ETR\&D, 50(2), 39-64 


\section{Maria Manuel Nascimento}

Professora Auxiliar

Universidade de Trás-os-Montes e Alto Douro (UTAD),

Vila Real, Portugal

Centro de Investigação em Didática e Tecnologia na Formação de Formadores (CIDTFF), Aveiro, Portugal

Email: mmsn@utad.pt

ORCID: 0000-0002-3913-4845

Paula Catarino

Professora Associada

Universidade de Trás-os-Montes e Alto Douro (UTAD), Vila Real, Portugal

Polo do Centro de Matemática da Universidade do Minho (CMAT-UTAD),

Vila Real, Portugal

Centro de Investigação em Didática e Tecnologia n a Formação de Formadores (CIDTFF), Aveiro, Portugal

Email: pcatarin@utad.pt

ORCID: 0000-0001-6917-5093

Eva Morais

Professora Auxiliar

Departamento de Matemática da Escola Ciências e Tecnologia Universidade de Trás-os-Montes e Alto Douro (UTAD), Vila Real, Portugal Polo do Centro de Matemática da Universidade do Minho (CMAT-UTAD), Vila Real, Portugal

Email: emorais@utad.pt ORCID: 0000-0002-3815-9821

Paulo Vasco

Professora Auxiliar

Departamento de Matemática da Escola Ciências e Tecnologia Universidade de Trás-os-Montes e Alto Douro, Vila Real, Portugal Polo do Centro de Matemática da Universidade do Minho (CMAT-UTAD), Vila Real, Portugal

Email: pvasco@utad.pt ORCID: 0000-0001-5460-4297

Correspondência

Maria Manuel Nascimento

Professora Auxiliar

Escola Ciências e Tecnologia

Universidade de Trás-os-Montes e Alto Douro

Quinta de Prados

5000-801 Vila Real

Data de submissão: Setembro 2018

Data de avaliação: Dezembro 2018

Data de publicação: Julho 2019 